\title{
Heat and Mass Transfer with Free Convection MHD Flow Past a Vertical Plate Embedded in a Porous Medium
}

\author{
Farhad Ali, Ilyas Khan, Sharidan Shafie, and Norzieha Musthapa \\ Department of Mathematics, Faculty of Science, Universiti Teknologi Malaysia (UTM), Skudai, 81310 Johor Bahru, Malaysia
}

Correspondence should be addressed to Sharidan Shafie; ridafie@yahoo.com

Received 7 November 2012; Accepted 11 April 2013

Academic Editor: Zhijun Zhang

Copyright (C) 2013 Farhad Ali et al. This is an open access article distributed under the Creative Commons Attribution License, which permits unrestricted use, distribution, and reproduction in any medium, provided the original work is properly cited.

An analysis to investigate the combined effects of heat and mass transfer on free convection unsteady magnetohydrodynamic (MHD) flow of viscous fluid embedded in a porous medium is presented. The flow in the fluid is induced due to uniform motion of the plate. The dimensionless coupled linear partial differential equations are solved by using Laplace transform method. The solutions that have been obtained are expressed in simple forms in terms of elementary function $\exp (\cdot)$ and complementary error function $\operatorname{erf} c(\cdot)$. They satisfy the governing equations; all imposed initial and boundary conditions and can immediately be reduced to their limiting solutions. The influence of various embedded flow parameters such as the Hartmann number, permeability parameter, Grashof number, dimensionless time, Prandtl number, chemical reaction parameter, Schmidt number, and Soret number is analyzed graphically. Numerical solutions for skin friction, Nusselt number, and Sherwood number are also obtained in tabular forms.

\section{Introduction}

The process of heat transfer or heat and mass transfer together occurs simultaneously in a moving fluid and plays an important role in the design of chemical processing equipment, nuclear reactors, and formation and dispersion of fog. A detailed discussion on this topic can be found in Raptis [1], Kim and Fedorov [2], El-Arabawy [3], Takhar et al. [4], Alam et al. [5], Chaudhary and Arpita [6], Ferdows et al. [7], Rajesh et al. [8], Rajesh and Varma [9], Bakr [10], and the references therein. Dass et al. [11] considered the mass transfer effects on flow past an impulsively started infinite isothermal vertical plate with constant mass flux. Muthucumaraswamy et al. [12] presented an exact solution to the problem of flow past an impulsively started infinite vertical plate in the presence of uniform heat and mass flux at the plate using Laplace transform technique.

Recently, the free convection flow of magnetohydrodynamic fluid has attracted many researchers in view of its numerous applications in geophysics, astrophysics, meteorology, aerodynamics, magnetohydrodynamic power generators and pumps, boundary layer controlenergy generators, accelerators, aerodynamics heating, polymer technology, petroleum industry, purification of crude oil, and in material processing such as extrusion, metal forming, continuous casting wire, and glass fibre drawing. Further, the convective flow through porous medium has applications in the field of chemical engineering for filtration and purification processes. In petroleum technology, it is used to study the movement of natural gas oil and water through oil channels or reservoirs, and in the field of agriculture engineering to study the underground water resources (see e.g., Hayat and Abbas [13], Rahman and Sattar [14], Kim [15], Kaviany [16], Vafai and Tien [17], Jha and Apere [18], Mandal et al. [19], Katagiri [20]). In view of such applications, Chaudhary and Jain [21] analyzed the magnetohydrodynamic free convection flow past an accelerated surface embedded in a porous medium and obtained the exact solutions for the velocity, temperature, and concentration fields using Laplace transform method. Seth et al. [22] investigated the unsteady MHD natural convection flow with radiative heat transfer past an impulsively moving plate with ramped wall temperature. Toki and Tokis [23] obtained the exact solutions for the unsteady free convection flows on a porous plate with time depending heating. Toki [24] developed the analytical solutions for free convection and mass transfer flow near a moving vertical porous plate. Das [25] developed the closed form solutions for the unsteady MHD free convection flow with thermal radiation and mass 
transfer over a moving vertical plate. In this continuation, the effect of heat mass transfer on unsteady MHD free convection flow past a moving vertical plate in a porous medium was investigated by Das and Jana [26]. They considered the impulsive, uniform, and oscillating motions of the plate with constant heat and mass diffusion and developed the exact solutions using Laplace transform technique. Recently, Osman et al. [27] analyzed the thermal radiation and chemical reaction effects on unsteady MHD free convection flow through a porous plate embedded in a porous medium with heat source/sink and the closed form solutions are obtained. Khan et al. [28] and Sparrow and Cess [29] analyzed the effects of Hall current and mass transfer on the unsteady MHD free convection flow in a porous channel. The motion in fluid is induced to the external pressure gradient and the closed form solutions for the velocity, temperature, and concentration fields are obtained.

Motivated by the above investigations the present paper aims to study the combined heat and mass effects on the unsteady MHD free convection flow of an incompressible viscous fluid passing through a porous medium. The flow in the fluid is caused due to the uniform motion of the plate. Exact solutions are derived for the velocity distributions, temperature, and concentration fields by using Laplace transform technique and presented graphically for small as well as large times. To the best of authors' knowledge this problem has not been studied before and the reported results are new. The present study is of course of great practical and technological importance, for example, in astrophysical regimes, the presence of planetary debris, cosmic dust, and so forth and creates a suspended porous medium saturated with plasma fluids. Combined buoyancy-generated heat and mass transfer, due to temperature and concentration variations with unsteady MHD free convection flow in fluid-saturated porous media, has several important applications in a variety of engineering processes including heat exchanger devices, petroleum reservoirs, chemical catalytic reactors, solar energy porous water collector systems, and ceramic materials.

This paper is organized as follows. A brief description of the problem formulation is given in Section 2. The exact solutions for the uniformly uniform motion of the plate are derived in Section 3. The graphical results and discussion are provided in Section 4. The conclusions of the paper are given in Section 5 whereas some future recommendations are included in Section 6.

\section{Description of the Problem Formulation}

Let us consider the unsteady one dimensional flow of an incompressible and electrically conducting viscous fluid caused due to the uniform motion of the plate. The $x^{*}$-axis is taken along the plate in the vertical direction and $y^{*}$-axis is taken normal to the plate. The electrically conducting fluid occupies the porous half space $y^{*}>0$. A uniform magnetic field $\mathbf{B}_{0}$ is acting in the transverse direction to the flow. The magnetic Reynolds number is assumed to be small and therefore the induced magnetic field is negligible compared with the applied magnetic field. The applied magnetic field is also taken weak so that Hall and ion slip effects may be neglected. Initially both the plate and fluid are at the same temperature $T_{\infty}^{*}$ and concentration $C_{\infty}^{*}$. At time $t=0^{+}$, the plate begins to slide in its own plane and accelerates against the gravitational field with uniform acceleration in $x^{*}$-direction. Then the temperature and concentration level are raised to $T_{w}^{*}$ and $C_{w}^{*}$ as shown in Figure 1 .

The Soret and thermal buoyancy effects are also considered. In addition to the above assumptions, we assume that the internal dissipation is absent and the usual Boussinesq approximation is taken into consideration. Moreover, the pressure gradient in the flow direction is compensated by the gradient of the hydrostatic pressure gradient of the fluid. As a result the governing equations of momentum, energy, and concentration are derived as follows:

$$
\begin{aligned}
\frac{\partial u^{*}}{\partial t^{*}}= & \nu \frac{\partial^{2} u^{*}}{\partial y^{* 2}}-\frac{\sigma B_{0}^{2} u^{*}}{\rho}-\frac{\nu u^{*}}{K^{*}} \\
& +g \beta\left(T^{*}-T_{\infty}^{*}\right)+g \beta^{*}\left(C^{*}-C_{\infty}^{*}\right), \\
\rho c_{p} \frac{\partial T^{*}}{\partial t^{*}}= & k \frac{\partial^{2} T^{*}}{\partial y^{* 2}}-\frac{\partial q_{r}^{*}}{\partial y^{*}}, \\
\frac{\partial C^{*}}{\partial t^{*}}= & D \frac{\partial^{2} C^{*}}{\partial y^{* 2}}+\frac{D K_{T}}{T_{m}} \frac{\partial^{2} T^{*}}{\partial y^{* 2}}-K_{r}^{*}\left(C^{*}-C_{\infty}^{*}\right),
\end{aligned}
$$

with the following initial and boundary conditions:

$$
\begin{gathered}
t^{*} \leq 0: u^{*}=0, \quad T^{*}=T_{\infty}^{*}, \quad C^{*}=C_{\infty}^{*}, \\
\forall y^{*} \geq 0, \\
t^{*} 0: u^{*}=f\left(t^{*}\right), \quad T^{*}=T_{w}^{*}, \quad C^{*}=C_{w}^{*} \\
\text { at } y^{*}=0, \\
: u^{*} \longrightarrow 0, \quad T^{*} \longrightarrow T_{\infty}^{*}, \quad C^{*} \longrightarrow C_{\infty}^{*} \\
\text { as } y^{*} \longrightarrow \infty
\end{gathered}
$$

where $f\left(t^{*}\right)$ is the uniform acceleration of the plate, $x^{*}$ and $y^{*}(\mathrm{~m})$ are the distances along and perpendicular to the plate, $t^{*}(\mathrm{~s})$ is the time, $u^{*}\left(\mathrm{~ms}^{-1}\right)$, denote the fluid velocity in the $x^{*}$-direction, $T^{*}(\mathrm{~K})$ temperature, $T_{\infty}^{*}(\mathrm{~K})$ temperature far from the plate, $T_{w}^{*}(\mathrm{~K})$ temperature at the wall, $C^{*}\left(\mathrm{~mol} \mathrm{~m}^{-3}\right)$ are the species concentration, $C_{w}^{*}\left(\mathrm{~mol} \mathrm{~m}^{-3}\right)$ surface concentration, $C_{\infty}^{*}\left(\mathrm{~mol} \mathrm{~m}^{-3}\right)$ species concentration far from the surface, $\beta(1 / \mathrm{K})$ the volumetric coefficient of thermal expansion, $\left.\beta^{*}(\mathrm{~mol} \mathrm{~m})^{-3}\right)^{-1}$ or $\left(\mathrm{m}^{3} \mathrm{~mol}^{-1}\right)$ is the volumetric coefficient of expansion for concentration, $v=$ $\mu / \rho\left(\mathrm{m}^{2} \mathrm{~s}^{-1}\right)$ the kinematic viscosity, $\mu\left(\mathrm{kgm}^{-1} \mathrm{~s}^{-1}\right)$ viscosity, $\rho\left(\mathrm{kgm}^{-3}\right)$ the fluid density, $c_{p}\left(\mathrm{~kg}^{-1} \mathrm{~K}^{-1}\right)$ is the specific heat capacity, $q_{r}^{*}$ the radiative heat flux in $x^{*}$-direction, $D\left(\mathrm{~m}^{2} \mathrm{~s}^{-1}\right)$ is mass diffusivity, $k\left(\mathrm{Wm}^{-1} \mathrm{~K}^{-1}\right)$ is the thermal conductivity of the fluid, $\sigma\left(\mathrm{Sm}^{-1}\right)$ the electrical conductivity of the fluid, $K^{*}>0\left(\mathrm{~m}^{2}\right)$ is the permeability of the porous medium, $T_{m}$ $(\mathrm{K})$ is the mean fluid temperature, $T_{\infty}^{*}$ is the free stream temperature, $C_{\infty}^{*}$ is the free stream concentration of the species, $K_{T}$ is the thermal-diffusion ratio, and $K_{r}^{*}$ the chemical reaction constant. The radiative heat flux term for an optically 


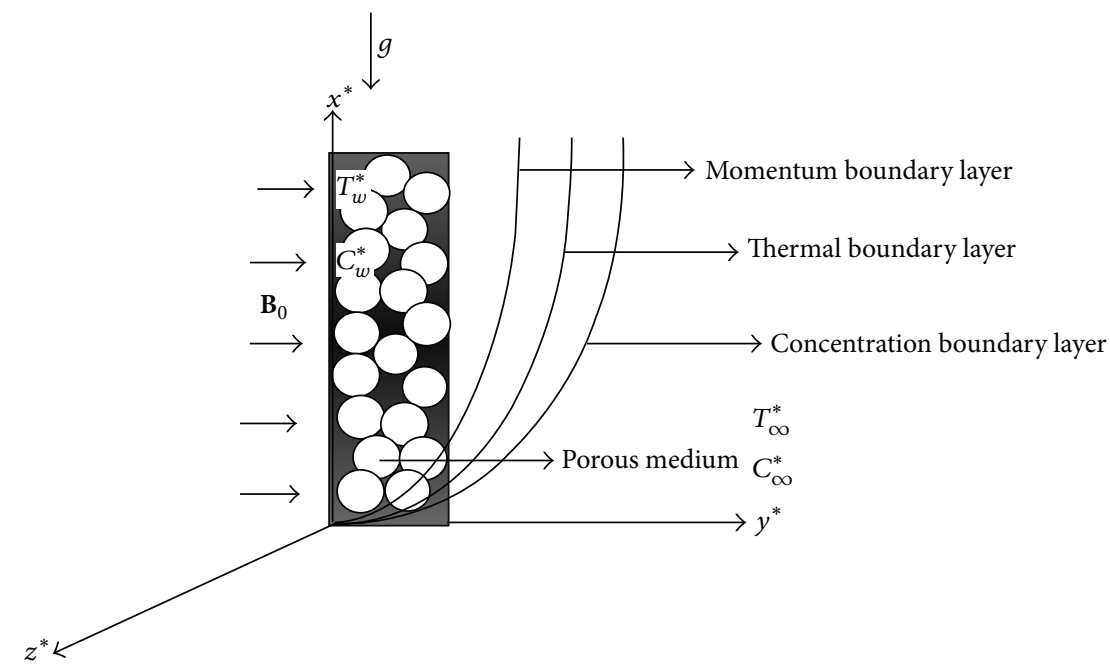

FIGURE 1: Flow geometry and physical coordinate system.

thin fluid is simplified by making use of the Rosseland approximation (Sparrow and Cess [29])

$$
q_{r}^{*}=-\frac{4 \sigma^{*}}{3 k^{*}} \frac{\partial T^{*^{4}}}{\partial y^{*}},
$$

where $\sigma^{*}\left(\mathrm{Wm}^{-2} \mathrm{~K}^{-4}\right)$ is the Stefan-Boltzmann constant and $k^{*}\left(\mathrm{~m}^{-1}\right)$ is the mean absorption coefficient. It is assumed that the temperature differences within the flow are sufficiently small such that the term $T^{*^{4}}$ is expressed as the linear function of temperature. Thus expanding $T^{*^{4}}$ about $T_{\infty}^{*}$ using Taylor series expansion and neglecting higher order terms we get

$$
T^{*^{4}} \cong 4 T_{\infty}^{*^{3}} T^{*}-3 T_{\infty}^{*^{4}} .
$$

From (5) and (6), (2) reduces to the following form:

$$
\rho c_{p} \frac{\partial T^{*}}{\partial t^{*}}=k \frac{\partial^{2} T^{*}}{\partial y^{* 2}}+\frac{16 \sigma^{*} T_{\infty}^{*^{3}}}{3 k^{*}} \frac{\partial^{2} T^{*}}{\partial y^{* 2}} .
$$

\section{Flow due to Uniform Motion of the Plate}

For uniform motion of the plate, we take $f\left(t^{*}\right)=A t^{*}$ and define the following dimensionless variables:

$$
\begin{gathered}
u=\frac{u^{*}}{(v A)^{1 / 3}}, \quad y=y^{*}\left(\frac{A}{\nu^{2}}\right)^{1 / 3}, \quad t=t^{*}\left(\frac{A^{2}}{v}\right)^{1 / 3}, \\
\theta=\frac{T^{*}-T_{\infty}^{*}}{T_{w}^{*}-T_{\infty}^{*}}, \quad \phi=\frac{C^{*}-C_{\infty}^{*}}{C_{w}^{*}-C_{\infty}^{*}},
\end{gathered}
$$

where $A$ with dimension $L / T^{2}$ denotes the uniform acceleration of the plate in $x$-direction, $u$ is the dimensionless velocity, $y$ dimensionless coordinate perpendicular to the plate, $t$ is the dimensionless time, $\theta$ is the dimensionless temperature and $\phi$ is the dimensionless species concentration.
Hence the governing equations in dimensionless form are

$$
\begin{gathered}
\frac{\partial u}{\partial t}=\frac{\partial^{2} u}{\partial y^{2}}-H u+\mathrm{Gr} \theta+\mathrm{Gm} \phi, \\
u(0, t)=t, \quad u(\infty, t)=0 \quad t>0, \\
u(y, 0)=0, \quad y \geq 0, \\
F^{*} \frac{\partial \theta}{\partial t}=\frac{\partial^{2} \theta}{\partial y^{2}}, \\
\theta(0, t)=1, \quad \theta(\infty, t)=0, \quad t>0, \\
\theta(y, 0)=0, \quad y \geq 0, \\
\frac{\partial \phi}{\partial t}=\frac{1}{\operatorname{Sc}} \frac{\partial^{2} \phi}{\partial y^{2}}+\operatorname{Sr} \frac{\partial^{2} \theta}{\partial y^{2}}-\gamma \phi, \\
\phi(0, t)=1, \quad \phi(\infty, t)=0, \quad t>0, \\
\phi(y, 0)=0, \quad y \geq 0,
\end{gathered}
$$

where

$$
\begin{gathered}
\frac{1}{K}=\frac{\nu^{4 / 3}}{K^{*} A^{2 / 3}}, \quad M^{2}=\frac{\sigma B_{0}^{2} \nu^{1 / 3}}{\rho A^{2 / 3}}, \\
\operatorname{Sr}=\frac{D K_{T}\left(T_{w}^{*}-T_{\infty}^{*}\right)}{T_{m} \nu\left(C_{w}^{*}-C_{\infty}^{*}\right)}, \quad \operatorname{Pr}=\frac{\mu c_{p}}{k}, \\
\mathrm{Gr}=\frac{g \beta\left(T_{w}^{*}-T_{\infty}^{*}\right)}{A}, \quad \mathrm{Gm}=\frac{g \beta^{*}\left(C_{w}^{*}-C_{\infty}^{*}\right)}{A}, \\
\gamma=\frac{K_{r}^{*} \nu^{1 / 3}}{A^{2 / 3}}, \\
H=M^{2}+\frac{1}{K}, \quad R=\frac{16 \sigma^{*} T_{\infty}^{*^{3}}}{3 k k^{*}}, \\
F^{*}=\frac{\operatorname{Pr}}{1+R}, \quad \mathrm{Sc}=\frac{\nu}{D} .
\end{gathered}
$$


Here $M$ is a magnetic parameter called Hartmann number, $K$ is the dimensionless permeability, $\mathrm{Sc}$ is Schmidt number, $R$ is Radiation parameter, Gr is Grashof number, and Sr is Soret number. The well-posed problems defined by (9)-(11) will be solved by using the Laplace transform technique. Hence the problem in the transformed plane is given as

$$
\begin{gathered}
\frac{d^{2} \bar{u}}{d y^{2}}-(H+q) \bar{u}+\mathrm{Gr} \bar{\theta}+\mathrm{Gm} \bar{\phi}=0, \\
\bar{u}(0, q)=\frac{1}{q^{2}}, \quad \bar{u}(\infty, q)=0, \\
\frac{d^{2} \bar{\theta}}{d y^{2}}-F^{*} q \bar{\theta}=0, \\
\bar{\theta}(0, q)=\frac{1}{q}, \quad \bar{\theta}(\infty, q)=0 \\
\frac{d^{2} \bar{\phi}}{d y^{2}}-(\gamma+q) \operatorname{Sc} \bar{\phi}+\operatorname{ScSr} \frac{d^{2} \bar{\theta}}{d y^{2}}=0 \\
\bar{\phi}(0, q)=\frac{1}{q}, \quad \bar{\phi}(\infty, q)=0
\end{gathered}
$$

where $q$ is the Laplace transformation parameter. by

The solutions of (13) in the transformed $q$-plane are given

$$
\begin{aligned}
& \bar{u}(y, q)=\frac{1}{q^{2}} \exp (-y \sqrt{q+H})+\frac{a_{8}}{q} \exp (-y \sqrt{q+H}) \\
& -\frac{a_{9}}{q+a_{0}} \exp (-y \sqrt{q+H}) \\
& +\frac{a_{10}}{q-H_{1}^{*}} \exp (-y \sqrt{q+H}) \\
& +\frac{a_{11}}{q-H^{*}} \exp (-y \sqrt{q+H}) \\
& +\frac{a_{5}}{q-H_{1}^{*}} \exp \left(-y \sqrt{F^{*} q}\right) \\
& -\frac{a_{11}}{q-H^{*}} \exp \left(-y \sqrt{F^{*} q}\right)+\frac{a_{4}}{q} \exp \left(-y \sqrt{F^{*} q}\right) \\
& -\frac{a_{6}}{q} \exp (-y \sqrt{(q+\gamma) \mathrm{Sc}}) \\
& +\frac{a_{9}}{q+a_{0}} \exp (-y \sqrt{(q+\gamma) \mathrm{Sc}}) \\
& -\frac{a_{7}}{q-H_{1}^{*}} \exp (-y \sqrt{(q+\gamma) \mathrm{Sc}}) \\
& \bar{\theta}(y, q)=\frac{1}{q} \exp \left(-y \sqrt{F^{*} q}\right)
\end{aligned}
$$

$$
\begin{aligned}
\bar{\phi}(y, q)= & \frac{1}{q} \exp (-y \sqrt{(q+\gamma) \mathrm{Sc}}) \\
& +\frac{S^{*}}{q-H_{1}^{*}} \exp (-y \sqrt{(q+\gamma) \mathrm{Sc}}) \\
& -\frac{S^{*}}{q-H_{1}^{*}} \exp \left(-y \sqrt{F^{*} q}\right),
\end{aligned}
$$

where

$$
\begin{aligned}
& \mathrm{Gr}^{*}=\frac{\mathrm{Gr}}{F^{*}-1}, \quad H^{*}=\frac{H}{F^{*}-1}, \\
& H_{1}^{*}=\frac{\gamma \mathrm{Sc}}{F^{*}-\mathrm{Sc}}, \quad S^{*}=\frac{\mathrm{ScSr} F^{*}}{F^{*}-S c}, \\
& a_{0}=\frac{\gamma \mathrm{Sc}-H}{\mathrm{Sc}-1}, \quad a_{1}=\frac{\mathrm{Gm}^{*}}{F^{*}-1}, \\
& a_{2}=\frac{\mathrm{Gm}}{\mathrm{Sc}-1}, \quad a_{3}=\frac{\mathrm{GmS}^{*}}{\mathrm{Sc}-1}, \quad a_{4}=\frac{\mathrm{Gr}^{*}}{H^{*}}, \\
& a_{5}=\frac{a_{1}}{H_{1}^{*}-H^{*}}, \quad a_{6}=\frac{a_{2}}{a_{0}}, \\
& a_{7}=\frac{a_{3}}{H_{1}^{*}+a_{0}}, \quad a_{8}=a_{6}-a_{4}, \quad a_{9}=a_{6}+a_{7}, \\
& a_{10}=a_{7}-a_{5}, \quad a_{11}=a_{4}+a_{5} .
\end{aligned}
$$

The inverse Laplace transform of (14)-(16) yields

$$
\begin{aligned}
& u(y, t)=I_{1}+a_{8} I_{2}-a_{9} I_{3}+a_{10} I_{4}+a_{11} I_{5}+a_{5} I_{6} \\
& -a_{11} I_{7}+a_{4} I_{8}-a_{6} I_{9}+a_{9} I_{10}-a_{7} I_{11} \text {, } \\
& \theta(y, t)=I_{8}, \\
& \phi(y, t)=S^{*}\left(I_{11}-I_{6}\right)+I_{9},
\end{aligned}
$$

with

$$
\begin{aligned}
& I_{1}=\frac{1}{2}\left[\left(t-\frac{y}{2 \sqrt{H}}\right) e^{-y \sqrt{H}} \operatorname{erf} c\left(\frac{y}{2 \sqrt{t}}-\sqrt{H t}\right)\right. \\
& \left.+e^{y \sqrt{H}} \operatorname{erf} c\left(\frac{y}{2 \sqrt{t}}+\sqrt{H t}\right)\left(t+\frac{y}{2 \sqrt{H}}\right)\right], \\
& I_{2}=\frac{1}{2}\left[e^{-y \sqrt{H}} \operatorname{erf} c\left(\frac{y}{2 \sqrt{t}}-\sqrt{H t}\right)\right. \\
& \left.+e^{y \sqrt{H}} \operatorname{erf} c\left(\frac{y}{2 \sqrt{t}}+\sqrt{H t}\right)\right], \\
& I_{3}=\frac{e^{-a_{0} t}}{2}\left[e^{-y \sqrt{H-a_{0}}} \operatorname{erf} c\left(\frac{y}{2 \sqrt{t}}-\sqrt{\left(H-a_{0}\right) t}\right)\right. \\
& \left.+e^{y \sqrt{H-a_{0}}} \operatorname{erf} c\left(\frac{y}{2 \sqrt{t}}+\sqrt{\left(H-a_{0}\right) t}\right)\right], \\
& I_{4}=\frac{e^{H_{1}^{*} t}}{2}\left[e^{-y \sqrt{H_{1}^{*}+H}} \operatorname{erf} c\left(\frac{y}{2 \sqrt{t}}-\sqrt{\left(H_{1}^{*}+H\right) t}\right)\right. \\
& \left.+e^{y \sqrt{H_{1}^{*}+H}} \operatorname{erf} c\left(\frac{y}{2 \sqrt{t}}+\sqrt{\left(H_{1}^{*}+H\right) t}\right)\right],
\end{aligned}
$$




$$
\begin{aligned}
& I_{5}=\frac{e^{H^{*} t}}{2}\left[e^{-y \sqrt{H^{*}+H}} \operatorname{erf} c\left(\frac{y}{2 \sqrt{t}}-\sqrt{\left(H+H^{*}\right) t}\right)\right. \\
& \left.+e^{y \sqrt{H^{*}+H}} \operatorname{erf} c\left(\frac{y}{2 \sqrt{t}}+\sqrt{\left(H+H^{*}\right) t}\right)\right], \\
& I_{6}=\frac{e^{H_{1}^{*} t}}{2}\left[e^{-y \sqrt{F^{*} H_{1}^{*}}} \operatorname{erf} c\left(\frac{y \sqrt{F^{*}}}{2 \sqrt{t}}-\sqrt{H_{1}^{*} t}\right)\right. \\
& \left.+e^{y \sqrt{F^{*} H_{1}^{*}}} \operatorname{erf} c\left(\frac{y \sqrt{F^{*}}}{2 \sqrt{t}}+\sqrt{H_{1}^{*} t}\right)\right], \\
& I_{7}=\frac{e^{H^{*} t}}{2}\left[e^{-y \sqrt{F^{*} H^{*}}} \operatorname{erf} c\left(\frac{y \sqrt{F^{*}}}{2 \sqrt{t}}-\sqrt{H^{*} t}\right)\right. \\
& \left.+e^{y \sqrt{F^{*} H^{*}}} \operatorname{erf} c\left(\frac{y \sqrt{F^{*}}}{2 \sqrt{t}}+\sqrt{H^{*} t}\right)\right], \\
& I_{8}=\operatorname{erf} c\left(\frac{y \sqrt{F^{*}}}{2 \sqrt{t}}\right) \\
& I_{9}=\frac{1}{2}\left[e^{-y \sqrt{\gamma \mathrm{Sc}}} \operatorname{erf} c\left(\frac{y \sqrt{\mathrm{Sc}}}{2 \sqrt{t}}-\sqrt{\gamma t}\right)\right. \\
& \left.+e^{y \sqrt{\gamma \mathrm{Sc}}} \operatorname{erf} c\left(\frac{y \sqrt{\mathrm{Sc}}}{2 \sqrt{t}}+\sqrt{\gamma t}\right)\right], \\
& I_{10}=\frac{e^{-a_{0} t}}{2}\left[e^{-y \sqrt{\mathrm{Sc}\left(\gamma-a_{0}\right)}} \operatorname{erf} c\left(\frac{y \sqrt{\mathrm{Sc}}}{2 \sqrt{t}}-\sqrt{\left(\gamma-a_{0}\right) t}\right)\right. \\
& \left.+e^{y \sqrt{S c\left(\gamma-a_{0}\right)}} \operatorname{erf} c\left(\frac{y \sqrt{S c}}{2 \sqrt{t}}+\sqrt{\left(\gamma-a_{0}\right) t}\right)\right], \\
& I_{11}=\frac{e^{H_{1}^{*} t}}{2}\left[e^{-y \sqrt{\operatorname{Sc}\left(H_{1}^{*}+\gamma\right)}} \operatorname{erf} c\left(\frac{y \sqrt{\mathrm{Sc}}}{2 \sqrt{t}}-\sqrt{\left(H_{1}^{*}+\gamma\right) t}\right)\right. \\
& \left.+e^{y \sqrt{\operatorname{sc}\left(H_{1}^{*}+\gamma\right)}} \operatorname{erf} c\left(\frac{y \sqrt{\mathrm{Sc}}}{2 \sqrt{t}}+\sqrt{\left(H_{1}^{*}+\gamma\right) t}\right)\right],
\end{aligned}
$$

where $\operatorname{erf} c(x)$ is the complementary error function. It is important to note that the above solutions are valid for $\operatorname{Pr} \neq 1$ and $S c \neq 1$. The solutions for $\operatorname{Pr}=1$ and $S c=1$, can be easily obtained by substituting $\operatorname{Pr}=\mathrm{Sc}=1$ into (10) and (11) and repeating the same process as discussed above.

3.1. Skin-Friction. The expression for skin-friction is given by

$$
\tau^{*}=-\left.\mu \frac{\partial u^{*}}{\partial y^{*}}\right|_{y^{*}=0}
$$

which in view of (8) reduces to

$$
\tau=-\frac{\partial u}{\partial y}, \quad \tau=\frac{\tau^{*}}{\rho U_{o}^{2}} .
$$

Hence from (18), we get

$$
\begin{aligned}
& \tau=\frac{a_{8} e^{-H t}}{\sqrt{\pi t}}-\frac{a_{11} \sqrt{F^{*}}}{\sqrt{\pi t}}+\frac{a_{4} \sqrt{F^{*}}}{\sqrt{\pi t}}+\frac{a_{5} \sqrt{F^{*}}}{\sqrt{\pi t}}-\frac{a_{6} e^{-\gamma t} \sqrt{S c}}{\sqrt{\pi t}} \\
& +\frac{e^{-H t} \sqrt{t}}{\sqrt{\pi}}+\frac{t \sqrt{H}}{2}(-1+\operatorname{erf}(\sqrt{H t}))+\frac{\operatorname{erf}(\sqrt{H t})}{2 \sqrt{H}} \\
& +a_{8} \sqrt{H} \operatorname{erf}(\sqrt{H t})+\frac{t \sqrt{H}}{2}(1+\operatorname{erf}(\sqrt{H t})) \\
& -a_{6} \sqrt{\operatorname{Sc} \gamma} \operatorname{erf}(\sqrt{\gamma t})-a_{11} e^{H^{*} t} \sqrt{H^{*} F^{*}} \operatorname{erf}\left(\sqrt{H^{*} t}\right) \\
& +a_{5} e^{H_{1}^{*} t} \sqrt{F^{*} H_{1}^{*}} \operatorname{erf}\left(\sqrt{H_{1}^{*} t}\right) \\
& -\frac{1}{2} a_{11} e^{H^{*} t}\left\{-\frac{2 e^{-H t-H^{*} t}}{\sqrt{\pi t}}+\sqrt{H+H^{*}}\right. \\
& \times\left(-1-\operatorname{erf}\left(\sqrt{\left(H+H^{*}\right) t}\right)+\sqrt{H+H^{*}}\right) \\
& \left.\times\left(1-\operatorname{erf}\left(\sqrt{\left(H+H^{*}\right) t}\right)\right)\right\} \\
& -\frac{1}{2} a_{10} e^{H_{1}^{*} t}\left\{-\frac{2 e^{-\left(H+H_{1}^{*}\right) t}}{\sqrt{\pi t}}+\sqrt{H+H_{1}^{*}}\right. \\
& \times\left(-1-\operatorname{erf}\left(\sqrt{\left(H+H_{1}^{*}\right) t}\right)\right) \\
& \left.+\sqrt{H+H_{1}^{*}}\left(1-\operatorname{erf}\left(\sqrt{\left(H+H_{1}^{*}\right) t}\right)\right)\right\} \\
& -\frac{1}{2} a_{9} e^{-a_{0} t}\left\{\frac{2 e^{a_{0} t-H t}}{\sqrt{\pi t}}-\sqrt{H-a_{0}}\right. \\
& \times\left(-1-\operatorname{erf}\left(\sqrt{-a_{0} t+H t}\right)\right) \\
& \left.-\sqrt{H-a_{0}}\left(1-\operatorname{erf}\left(\sqrt{-a_{0} t+H t}\right)\right)\right\}
\end{aligned}
$$

$$
\begin{aligned}
-\frac{1}{2} a_{9} e^{-a_{0} t}\{ & -\frac{2 e^{a_{0} t-\gamma t} \sqrt{\mathrm{Sc}}}{\sqrt{\pi t}}+\sqrt{\left(-a_{0}+\gamma\right) \mathrm{Sc}} \\
& \times\left(-1-\operatorname{erf}\left(\sqrt{\left(-a_{0}+\gamma\right) t}\right)\right) \\
& +\sqrt{\left(-a_{0}+\gamma\right) \mathrm{Sc}} \\
& \left.\times\left(1-\operatorname{erf}\left(\sqrt{\left(-a_{0}+\gamma\right) t}\right)\right)\right\}
\end{aligned}
$$$$
-\frac{1}{2} a_{7} e^{H_{1}^{*} t}\left\{\frac{2 e^{-\left(H_{1}^{*}+\gamma\right) t}}{\sqrt{\pi t}}-\sqrt{\mathrm{Sc}} \sqrt{H_{1}^{*}+\gamma}\right.
$$

$$
\times\left(-1-\operatorname{erf}\left(\sqrt{\left(H_{1}^{*}+\gamma\right) t}\right)\right)
$$




$$
\begin{aligned}
& -\sqrt{\mathrm{Sc}} \sqrt{H_{1}^{*}+\gamma} \\
& \left.\times\left(1-\operatorname{erf}\left(\sqrt{\left(H_{1}^{*}+\gamma\right) t}\right)\right)\right\} .
\end{aligned}
$$

3.2. Nusselt Number. The rate of heat transfer for the present problem is given as

$$
\begin{gathered}
\mathrm{Nu}=-\left.k \frac{\partial T^{*}}{\partial y^{*}}\right|_{y^{*}=0}, \\
\mathrm{Nu}=\left.\frac{\partial \theta}{\partial y}\right|_{y^{*}=0}, \\
\mathrm{Nu}=\sqrt{\frac{F^{*}}{\pi t}} .
\end{gathered}
$$

3.3. Sherwood Number. The rate of mass transfer is given by

$$
\begin{gathered}
\mathrm{Sh}=\left.\frac{\partial C^{*}}{\partial y^{*}}\right|_{y^{*}=0}, \\
\mathrm{Sh}=-\frac{1}{\sqrt{\pi t}}\left\{e ^ { - \gamma t } \left(-e^{\gamma t} \sqrt{F^{*}} S^{*}+\sqrt{\mathrm{Sc}}+S^{*} \sqrt{\mathrm{Sc}}\right.\right. \\
\left.-e^{H^{*} t+\gamma t} S^{*} \sqrt{\pi t H^{*} F^{*}} \operatorname{erf}\left(\sqrt{H^{*} t}\right)\right) \\
+e^{\gamma t} \sqrt{\gamma \mathrm{Sc} \pi t} \operatorname{erf}(\sqrt{\gamma t})+e^{H^{*} t+\gamma t} S^{*} \\
\left.\times \sqrt{\pi t \mathrm{Sc}} \sqrt{H^{*}+\gamma} \operatorname{erf}\left(\sqrt{\left(H^{*}+\gamma\right) t}\right)\right\} .
\end{gathered}
$$

It is important to note that solutions (18)-(20) satisfy all the imposed boundary and initial conditions. Further, the solutions obtained here are more general and the existing solutions in the literature appeared as the limiting cases.

(1) The present solutions given by (18)-(20) in the absence of radiation effect and by taking the thermaldiffusion ratio $\left(K_{T}\right)$ and the chemical reaction constant $\left(K_{r}^{*}\right)$ equal to zero reduce to the solutions of Das and Jana [26] (see (4.2), (3.8), and (3.9)).

(2) The solutions (18)-(20) for the flow of optically thick fluid in a nonporous medium with $K_{r}^{*}=K_{T}=0$, give the solutions of Das [25] (see (4.4), (3.1), and (3.2)).

\section{Graphical Results and Discussion}

An exact analysis is presented to investigate the combined effects of heat mass transfer on the transient MHD free convective flow of an incompressible viscous fluid past a vertical plate moving with uniform motion and embedded in a porous medium. The expressions for the velocity $u$, temperature $\theta$, and concentration $\phi$ are obtained by using Laplace transform method. In order to understand the physical behavior of the dimensionless parameters such as Hartmann

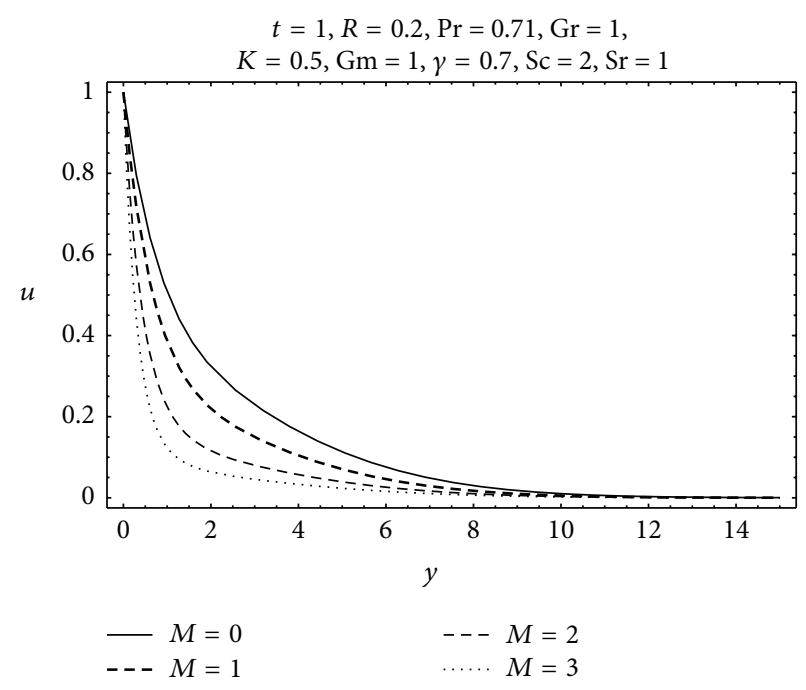

FIgURE 2: Velocity profiles for different values of $M$.

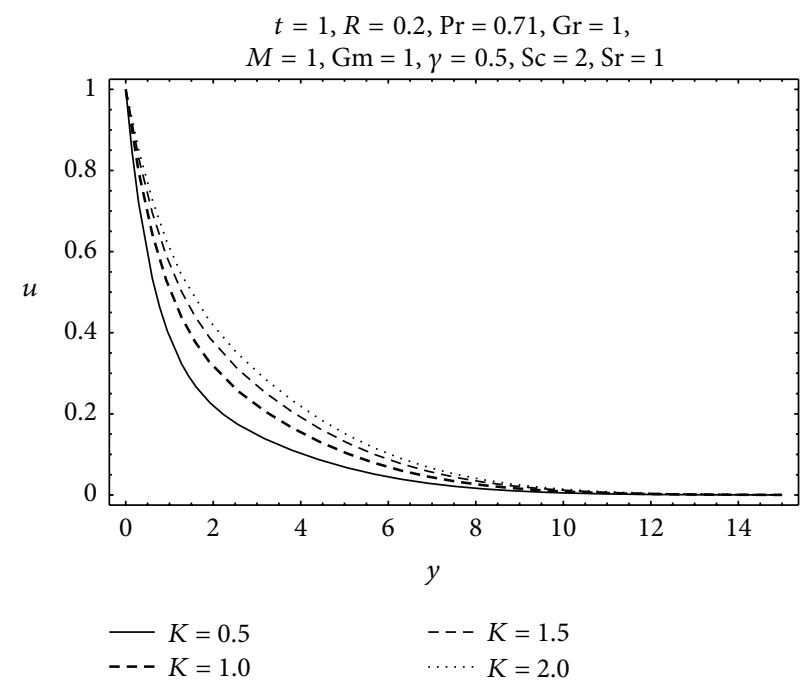

FIGURE 3: Velocity profiles for different values of $K$.

number $M$ also called magnetic parameter, permeability parameter $K$, Grashof number Gr, dimensionless time $t$, Prandtl number Pr, radiation parameter $R$, chemical reaction parameter $\gamma$, Schmidt number Sc, and Soret number Sr, Figures 2-17 have been displayed for $u, \theta$, and $\phi$.

Figure 2 presents the velocity profile for different values of $M$. It is observed that the velocity and boundary layer thickness decreases upon increasing the Hartmann number $M$. It is due to the fact that the application of transverse magnetic field results a resistive type force (called Lorentz force) similar to drag force and upon increasing the values of $M$ increases the drag force which leads to the deceleration of the flow. Figure 3 is sketched in order to explore the variations of permeability parameter $K$. It is found that the velocity increases with increasing values of $K$. This is due to the fact that increasing values of $K$ reduces the drag force which assists the fluid considerably to move fast. The variation of 


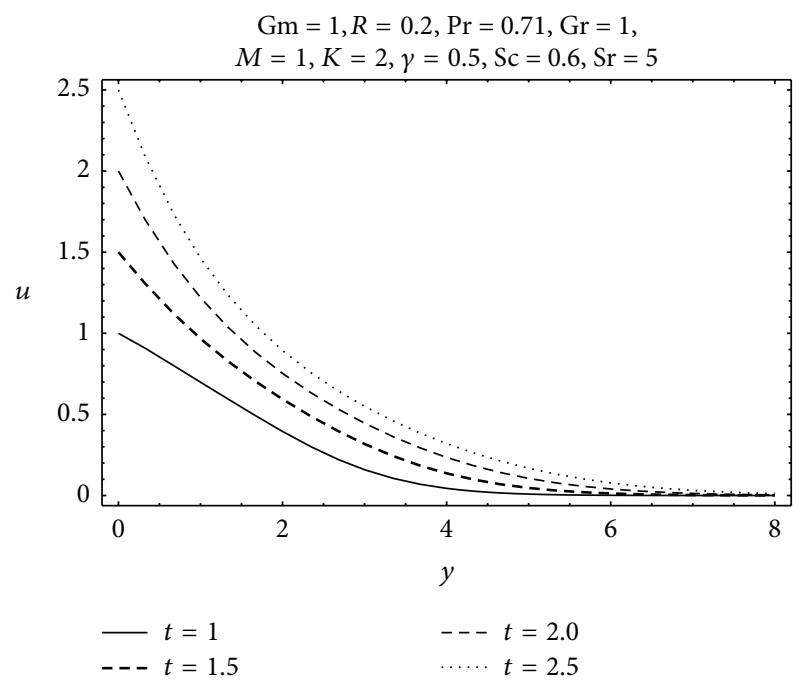

FIGURE 4: Velocity profiles for different values of $t$.

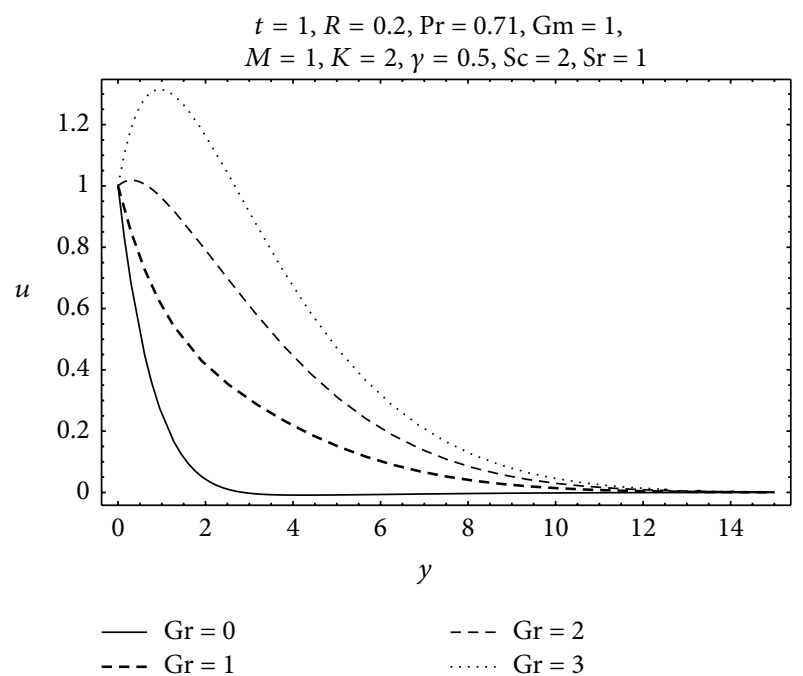

FIGURE 5: Velocity profiles for different values of Gr.

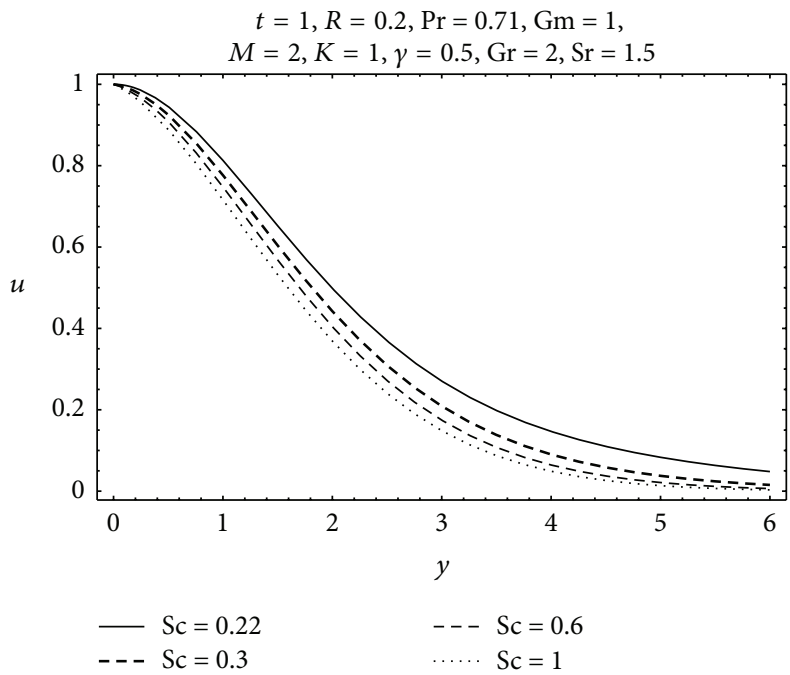

FIGURE 6: Velocity profiles for different values of Sc.

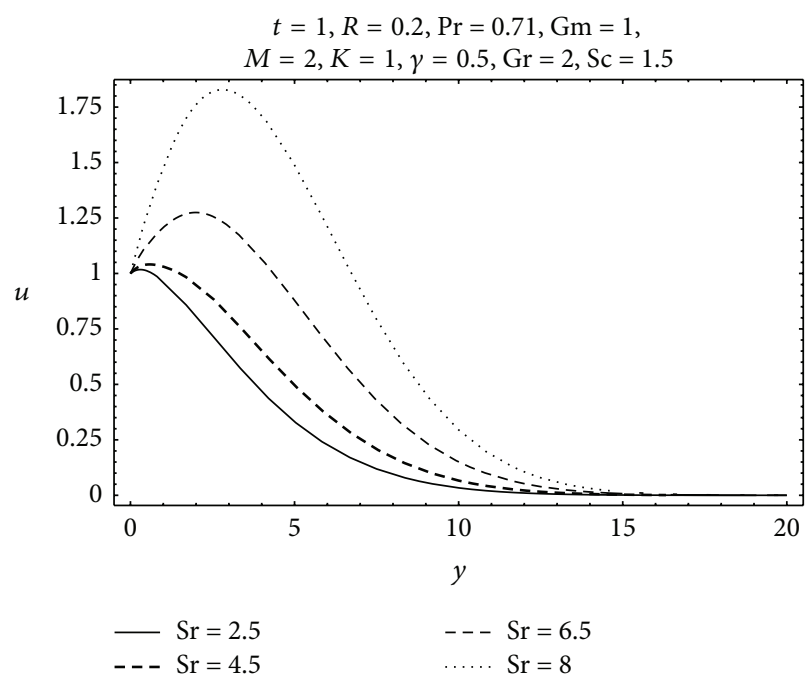

FIGURE 7: Velocity profiles for different values of Sr.

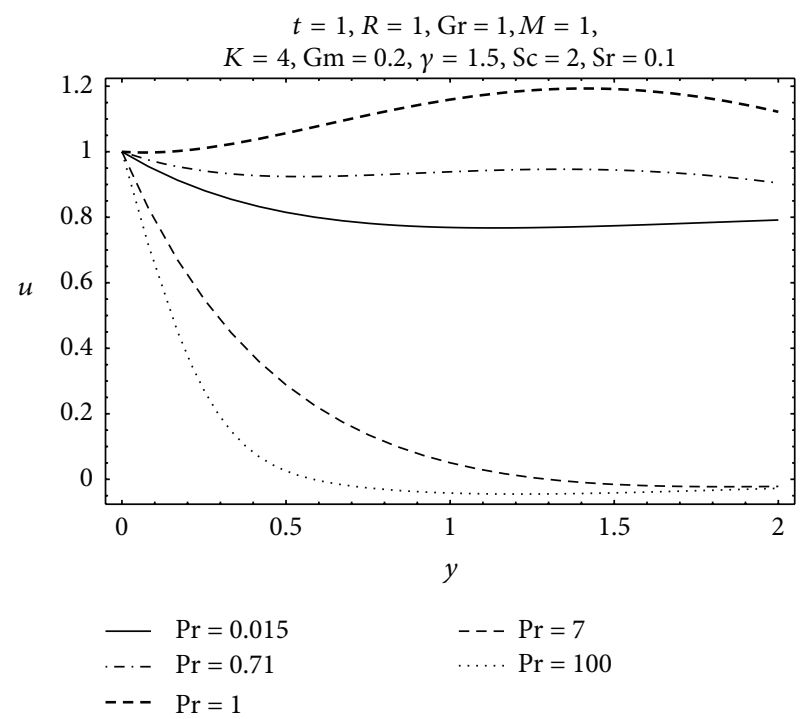

FIGURE 8: Velocity profiles for different values of Pr.

velocity for different values of dimensionless time $t$ is shown in Figure 4. It is noticed that velocity increases with increasing time. Further, this figure verifies the boundary conditions of velocity given in (9). Initially, velocity takes the values of time and later for large values of $y$, and the velocity tends to zero with increasing time. It is observed from Figure 5 that the fluid velocity increases with increasing Gr. Figure 6 reveals that velocity profiles decrease with the increase of Schmidt number Sc, while an opposite phenomenon is observed in case of Soret number Sr as shown in Figure 7.

Velocity, temperature, and concentration profiles for some realistic values of Prandtl number $\operatorname{Pr}=0.015,0.71,1.0$, $7.0,100$, which are important in the sense that they physically correspond to mercury, air, electrolytic solution, water, and engine oil, are shown in Figures 8-10, respectively. From Figure 8 , it is found that the momentum boundary layer thickness increases for the fluids with $\operatorname{Pr}<1$ and decreases for 


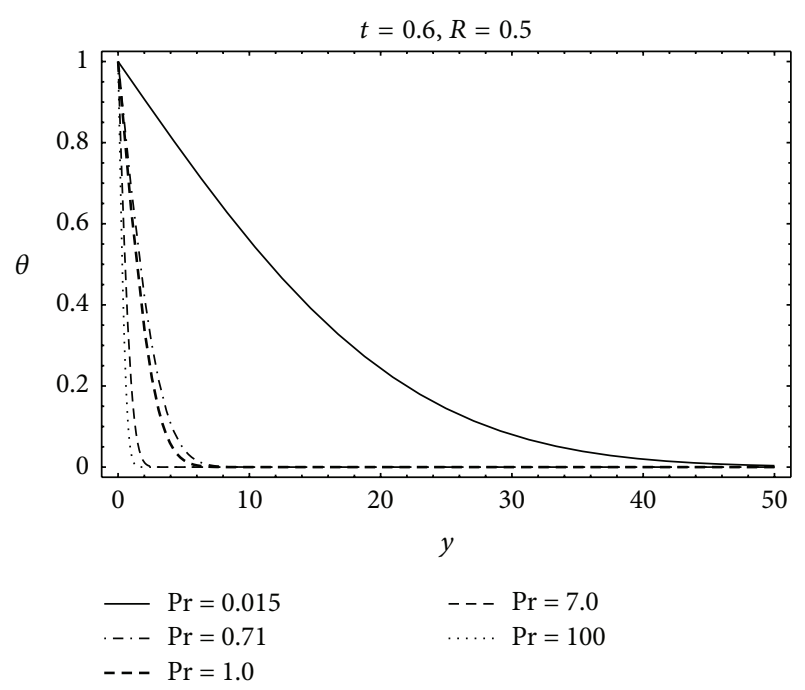

FIgURE 9: Temperature profiles for different values of Pr.

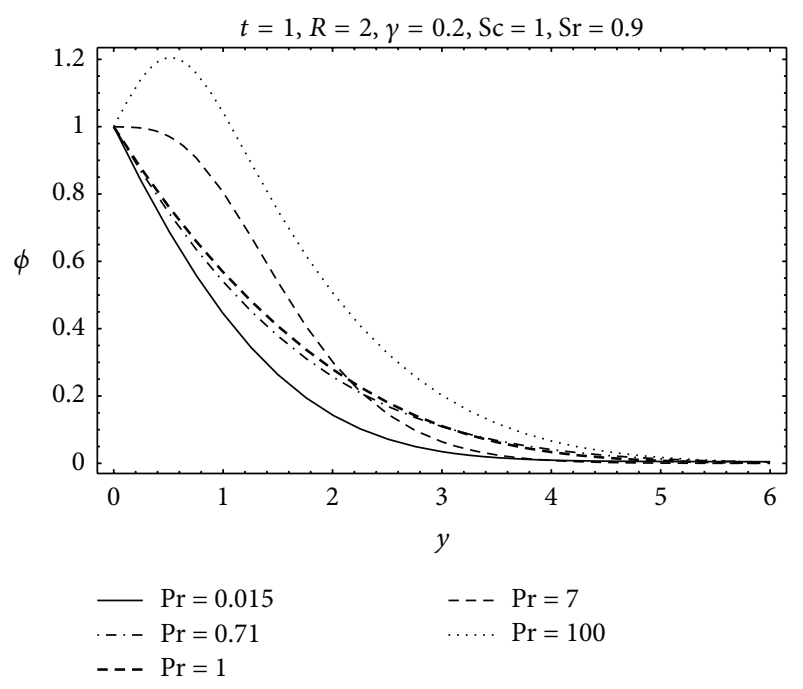

FIgURE 10: Concentration profiles for different values of Pr.

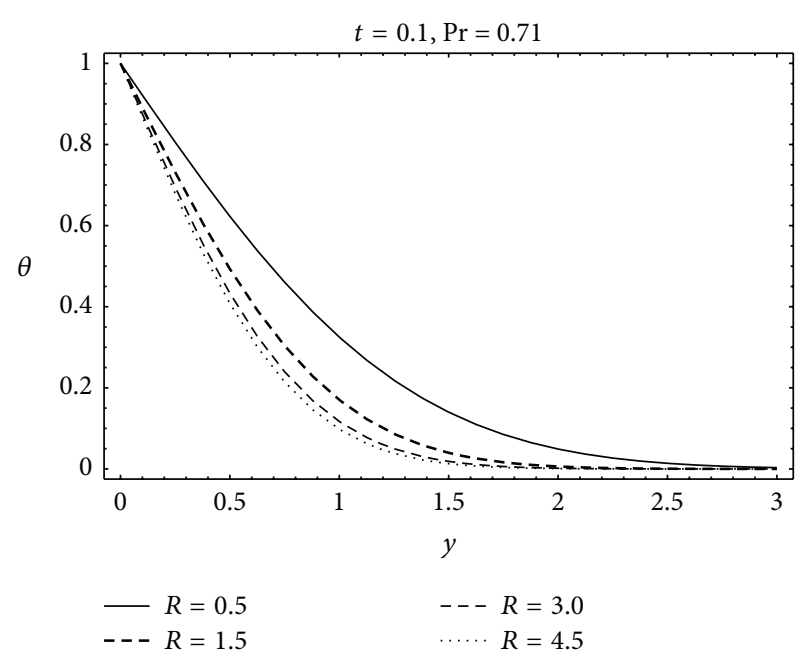

FIgURE 11: Temperature profiles for different values of $R$.

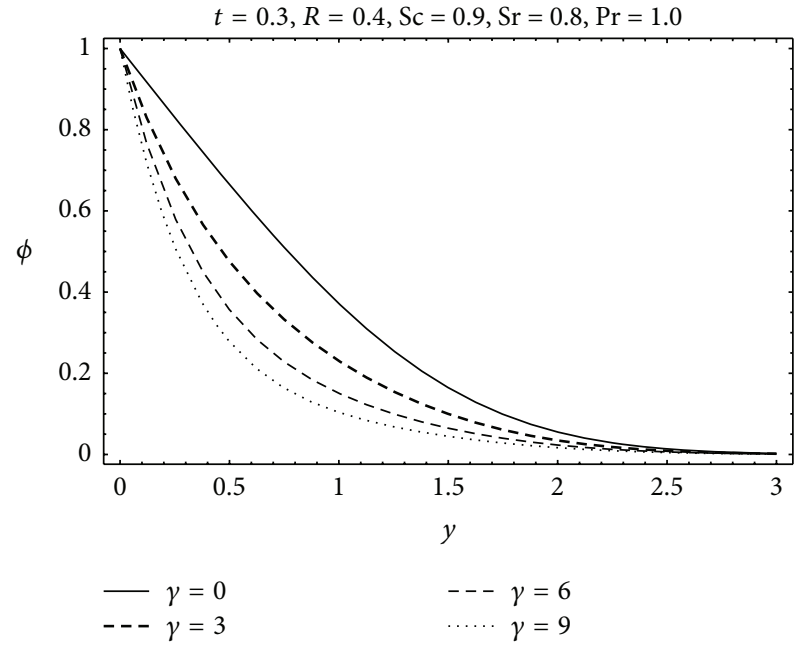

FIGURE 12: Concentration profiles for different values of $\gamma$.

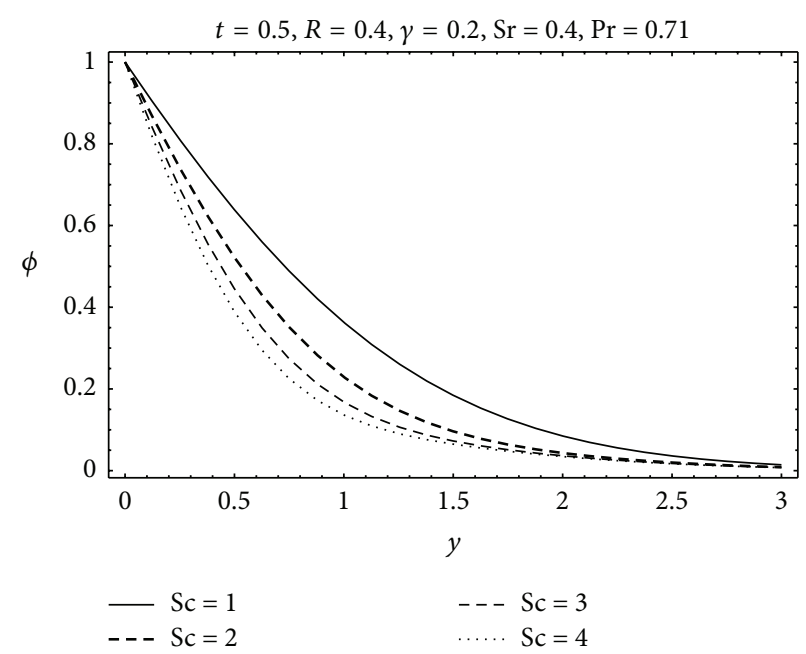

FIgURE 13: Concentration profiles for different values of Sc.

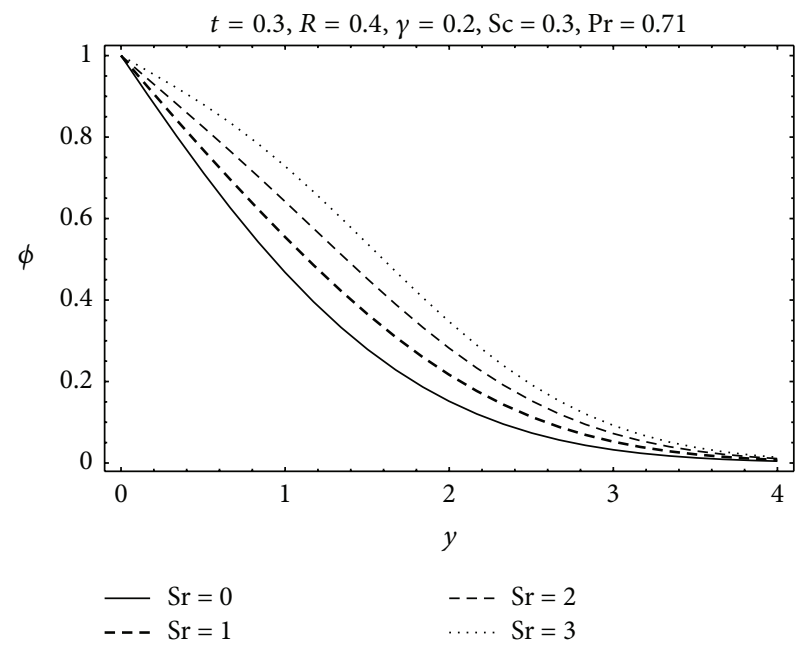

FIGURE 14: Concentration profiles for different values of Sr. 


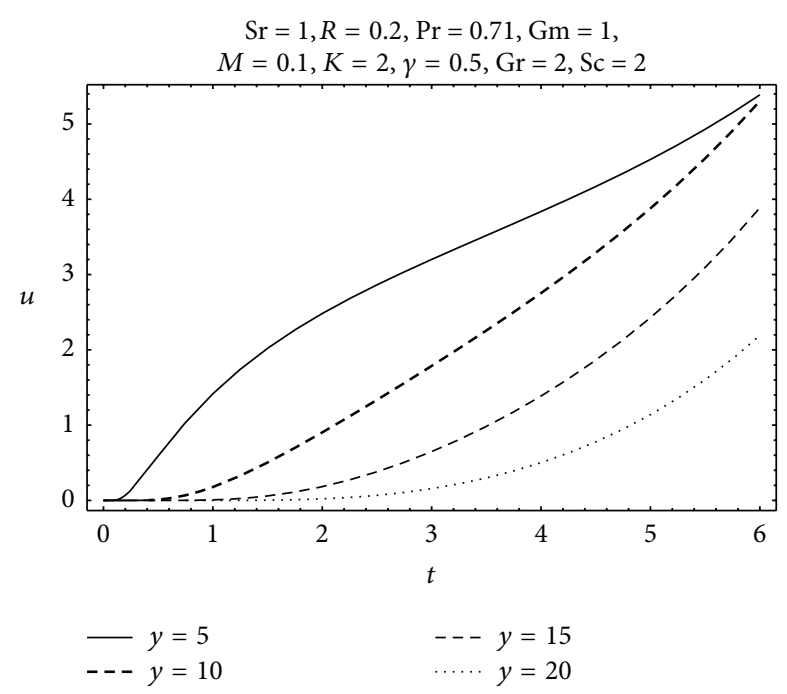

FIGURE 15: Velocity profiles for different values of $y$.

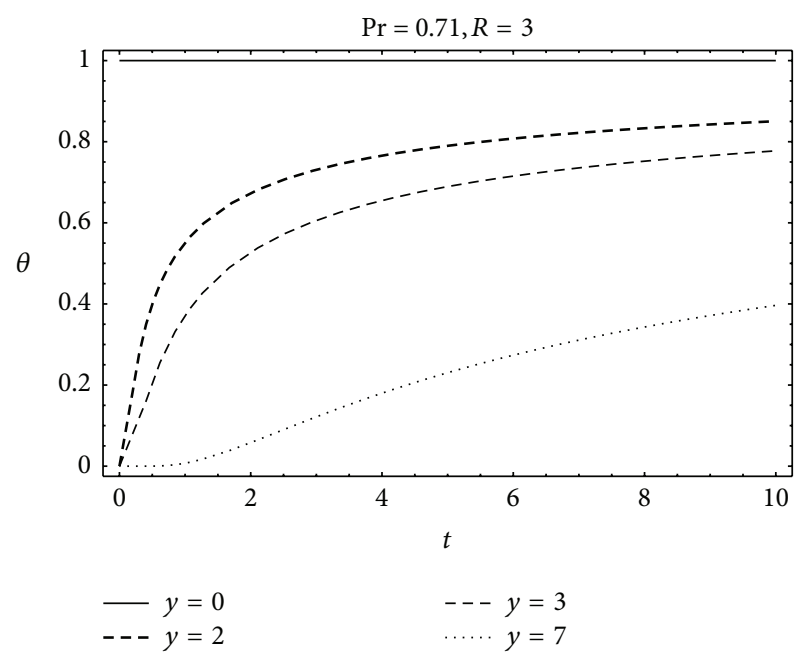

FIGURE 16: Temperature profiles for different values of $y$.

$\operatorname{Pr}>1$. The Prandtl number actually describes the relationship between momentum diffusivity and thermal diffusivity and hence controls the relative thickness of the momentum and thermal boundary layers. When $\mathrm{Pr}$ is small, that is, $\mathrm{Pr}=$ 0.015 , it is noticed that the heat diffuses very quickly compared to the velocity (momentum). This means that for liquid metals the thickness of the thermal boundary layer is much bigger than the velocity boundary layer.

In Figure 9, we observe that the temperature decreases with increasing values of Prandtl number Pr. It is also observed that the thermal boundary layer thickness is maximum near the plate and decreases with increasing distances from the leading edge and finally approaches to zero. Furthermore, it is noticed that the thermal boundary layer for mercury which corresponds to $\operatorname{Pr}=0.015$ is greater than those for air, electrolytic solution, water, and engine oil. It is justified due to the fact that thermal conductivity of fluid decreases with increasing Prandtl number Pr and hence

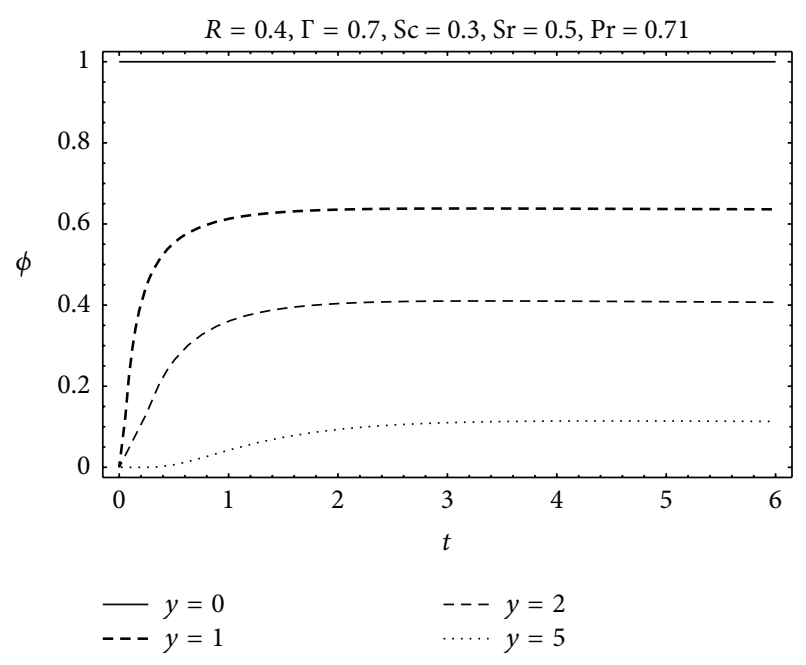

FIGURE 17: Concentration profiles for different values of $y$.

decreases the thermal boundary layer thickness and the temperature profiles. We observed from Figure 10 that the concentration of the fluid increases for large values of Prandtl number Pr.

The effects of radiation parameter $R$ on the temperature profiles are shown in Figure 11. It is found that the temperature profiles $\theta$, being as a decreasing function of $R$, decelerate the flow and reduce the fluid velocity. Such an effect may also be expected, as increasing radiation parameter $R$ makes the fluid thick and ultimately causes the temperature and the thermal boundary layer thickness to decrease. The influence of $\gamma, \mathrm{Sc}$, and $\mathrm{Sr}$ on the concentration profiles $\phi$ is shown in Figures 12-14. It is depicted from Figures 12 and 13 that the increasing values of $\gamma$ and Sc lead to fall in the concentration profiles. Figure 14 depicts that the concentration profiles increase when Soret number Sr is increased. Furthermore, we observe that in the absence of Soret effects, the concentration profile tends to a steady state in terms of $y$; this may be seen from (11). When Soret effects are present, then at large times, the solutal solution consists of this steady-state solution and an evolving "particular integral" due to the presence of the temperature term.

An important aspect of the unsteady problem is that it describes the flow situation for small times $(t \ll 1)$ as well as large times $(t \rightarrow \infty)$. Therefore, the present solutions for velocity distributions, temperature, and concentration profiles are displayed for both small and large times (see Figures 15-17). The velocity versus time graph for different values of independent variable $y$ is plotted in Figure 15. It is found from Figure 15 that the velocity decreases as independent variable $y$ increases. Further, it is interesting to note that initially, when $t=0$ the fluid velocity is zero which is also true from the initial condition given in (9). However, it is observed that as time increases, the velocity increases and after some time of initiation, this transition stops and the fluid motion becomes independent of time and hence the solutions are called steady-state solutions. This transition is smooth as we can see from the graph. On the other hand, from the velocity 


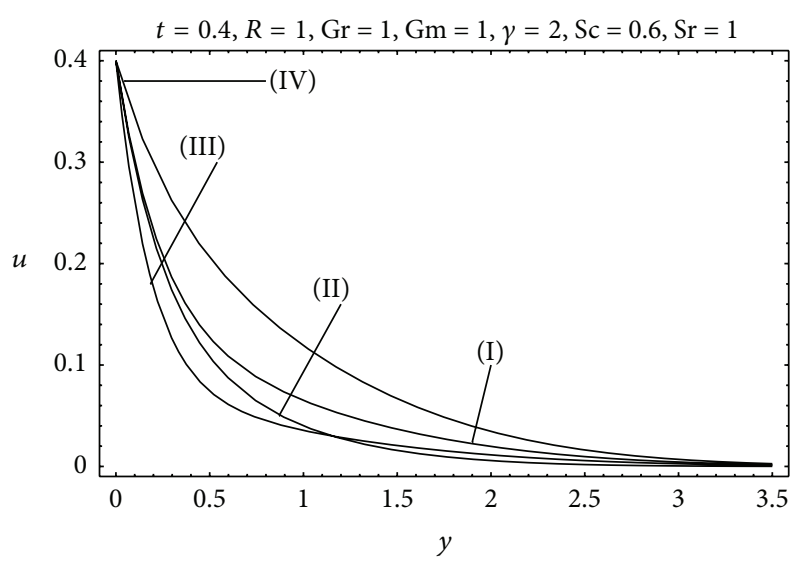

$\begin{array}{rrrrrrrrr}\text { Curves } & \operatorname{Pr} & M & K & \text { Curves } & \operatorname{Pr} & M & K \\ \text { (I) } & 0.71 & 2 & 0.1 & \text { (III) } & 0.71 & 4 & 0.1 \\ \text { (II) } & 7 & 2 & 0.1 & \text { (IV) } & 0.71 & 2 & 0.4\end{array}$

Figure 18: Combined effect of various parameters on velocity profiles.

versus time graph for different values of the independent variable $y$ (see Figure 15), it is found that the velocity at $y=0$, is maximum and continuously decreases for large values of $y$. It is further noted from this figure that for large values of $y$, that is, when $y \rightarrow \infty$, the velocity profile approaches to zero. A similar behavior was also expected in view of the boundary conditions given in (9). Hence this figure shows the correctness for the obtained analytical result given by (18).

Similarly, the next two Figures 16 and 17 are plotted to describe the transient and steady-state solutions which include the effects of heating and mass diffusion. It is clear from Figure 16 that the dimensionless temperature $\theta$ has its maximum value unity at $y=0$ and then decreasing for further large values of $y$ and ultimately approaches to zero. A similar behavior was also expected due the fact that the temperature profile is 1 for $y=0$ and for large values of $y$, its value approaches to 0 , which is mathematically true in view of the boundary conditions given in (10). From Figure 17, it is depicted that the variation of time on the concentration profile presents similar results as for the temperature profile in qualitative sense. However, these results are not the same quantitatively.

A very important phenomenon to see the combined effects of the embedded flow parameters on the velocity, temperature, and concentration profiles is analyzed in Figures 1821. Figure 18 is plotted to observe the combined effects of $\mathrm{Pr}$, $M$, and $K$ on velocity in case of cooling of the plate $(\mathrm{Gr}>0)$ as shown by Curves I-IV. Curves I \& II are sketched to display the effects of $\mathrm{Pr}$ on velocity. The values of Prandtl number are chosen as $\operatorname{Pr}=0.71$ (air) and $\operatorname{Pr}=7$ (water), which are the most encountered fluids in nature and frequently used in engineering and industry. We can from the comparison of Curves I \& II that velocity decreases upon increasing Prandtl number Pr. Curves I \& III present the influence of Hartmann number $M$ on velocity profiles. It is clear from

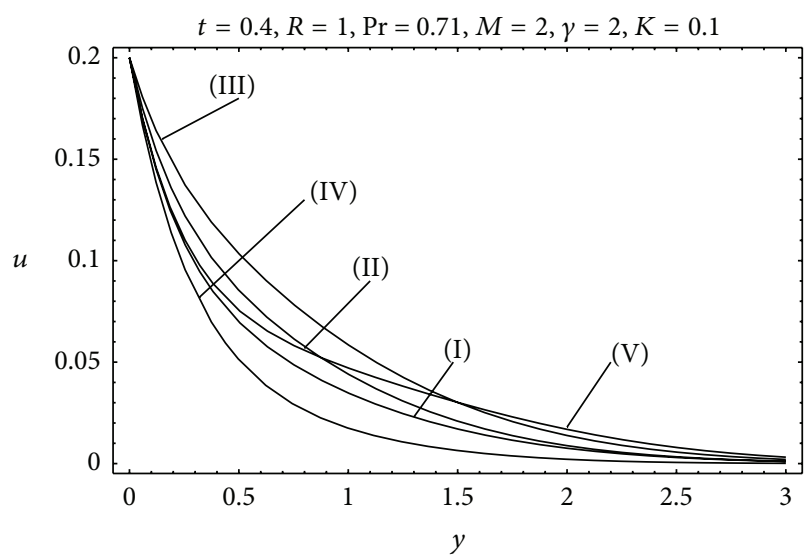

$$
\begin{array}{rccccrcccc}
\text { Curves } & \text { Gr } & \text { Gm } & \text { Sc } & \text { Sr } & \text { Curves } & \text { Gr } & \text { Gm } & \text { Sc } & \text { Sr } \\
\text { (I) } & 0.5 & 1 & 0.22 & 1 & \text { (IV) } & 0.5 & 1 & 0.6 & 1 \\
\text { (II) } & 1 & 1 & 0.22 & 1 & \text { (V) } & 0.5 & 1 & 0.22 & 2 \\
\text { (III) } & 0.5 & 2 & 0.22 & 1 & & & & &
\end{array}
$$

FIGURE 19: Combined effect of various parameters on velocity profiles.

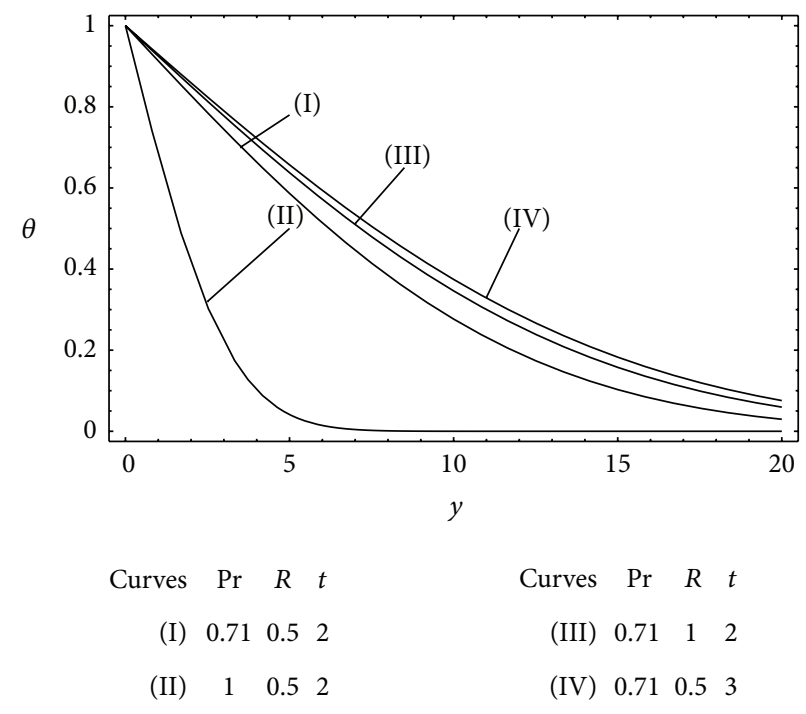

Figure 20: Combined effect of various parameters on temperature profiles.

these curves that velocity decreases when $M$ is increased. The effect of permeability parameter $K$ on the velocity is quite different to that of $M$. This fact is shown from the comparison of Curves I \& IV. Figure 19 is plotted to show the effects of Grashof number Gr, modified Grashof number $\mathrm{Gm}$, Schmidth number Sc, and Soret number Sr on velocity profiles. Curves I \& II show that velocity increases when Gr is increased. It is observed that the effect of $\mathrm{Gm}$ on velocity is the same as Gr. This fact is shown from the comparison of Curves I \& III. The effect of Sc on velocity is shown from the comparison of Curves I \& IV. Here we choose the Schmidth number values as $\mathrm{Sc}=0.22$ and $\mathrm{Sc}=0.6$ which physically 


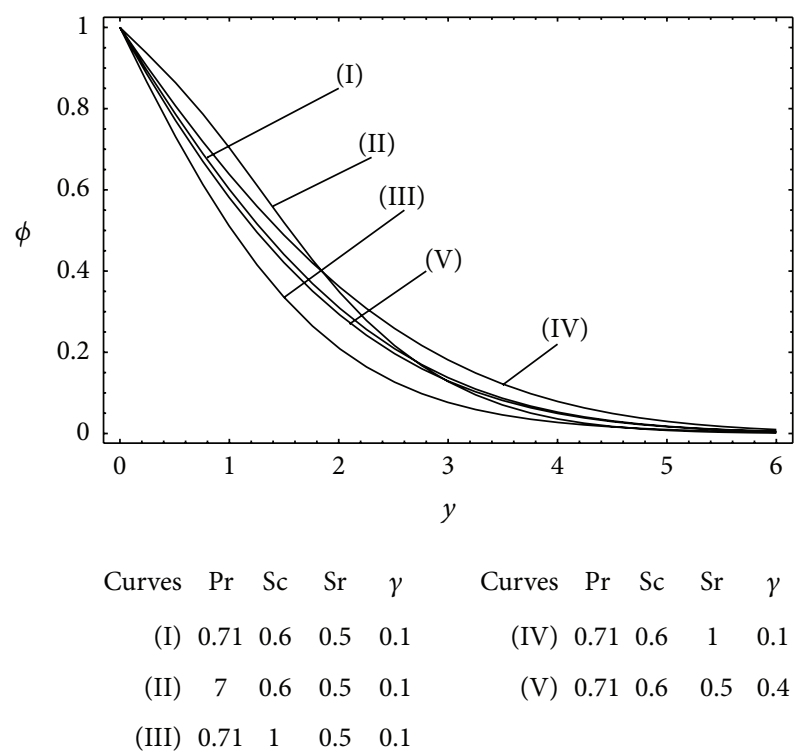

FIGURE 21: Combined effect of various parameters on concentration profiles.

correspond to Helium and water vapours, respectively. From these curves it is clear that velocity decreases when Sc is increased. The effect of Soret number Sr on velocity is quite opposite to that of Sc.

Figure 20 is plotted to show the effects of Prandtl number $\operatorname{Pr}$, radiation parameter $R$, and time $t$ on the temperature profiles. The comparison of Curves I \& II shows the effects of Pr on the temperature profiles. Two different values of Prandtl number Pr, namely, $\operatorname{Pr}=0.71$ and $\operatorname{Pr}=1$ corresponding to air and electrolyte are chosen. It is observed that temperature decreases with increasing Pr. Furthermore, the temperature profiles for increasing values of radiation parameter $R$ indicate an increasing behavior as shown in Curves I \& III. A behavior was expected because the radiation parameter $R$ signifies the relative contribution of conduction heat transfer to thermal radiation transfer. The effect of time $t$ on temperature is the same as observed for radiation. This fact is shown from the comparison of Curves I \& IV. Graphical results of concentration profiles for different values of Prandtl number Pr, Schmidth number Sc, Soret number Sr, and chemical reaction parameter $\gamma$ are shown in Figure 21. Comparison of Curves I \& II shows that concentration profiles increase for the increasing values of Pr. The effect of Sc on the concentration is shown from the comparison of Curves I \& III. Here we choose real values for Schmidth number as $\mathrm{Sc}=0.6$ and $\mathrm{Sc}=1$ which physically correspond to water vapours and methanol. It is observed that an increase in Sc decreases the concentration. The effect of Soret number Sr on the concentration is seen from the comparison of Curves I \& IV. It is observed that concentration increases when $\mathrm{Sr}$ increases. The effect of chemical reaction parameter $\gamma$ on the concentration is quite opposite to that of Sr. This fact is shown from the comparison of Curves I \& V.

The numerical values of the skin friction $(\tau)$, Nusselt number $(\mathrm{Nu})$, and Sherwood number $(\mathrm{Sh})$ are computed in
TABLE 1: The effects of various parameters on skin friction $(\tau)$ when $t=1, R=0.2, \gamma=0.7$.

\begin{tabular}{lccccccc}
\hline $\operatorname{Pr}$ & $M$ & $K$ & $\mathrm{Gr}$ & $\mathrm{Sc}$ & $\mathrm{Sr}$ & $\mathrm{Gm}$ & $\tau$ \\
\hline 0.71 & 1 & 0.5 & 1 & 2 & 1 & 1 & 1.27 \\
$\mathbf{1}$ & 1 & 0.5 & 1 & 2 & 1 & 1 & 1.30 \\
0.71 & $\mathbf{2}$ & 0.5 & 1 & 2 & 1 & 1 & 2.05 \\
0.71 & 1 & $\mathbf{1}$ & 1 & 2 & 1 & 1 & 0.94 \\
0.71 & 1 & 0.5 & $\mathbf{2}$ & 2 & 1 & 1 & 0.85 \\
0.71 & 1 & 0.5 & 1 & $\mathbf{3}$ & 1 & 1 & 1.31 \\
0.71 & 1 & 0.5 & 1 & 2 & 3 & 1 & 1.25 \\
0.71 & 1 & 0.5 & 1 & 2 & 1 & $\mathbf{2}$ & 0.96 \\
\hline
\end{tabular}

TABLE 2: The effects of various parameters on Nusselt number $(\mathrm{Nu})$ when $t=1$.

\begin{tabular}{lcc}
\hline $\operatorname{Pr}$ & $R$ & $\mathrm{Nu}$ \\
\hline 0.71 & 0.2 & 0.43 \\
$\mathbf{1}$ & 0.2 & 0.51 \\
0.71 & $\mathbf{0 . 4}$ & 0.40 \\
\hline
\end{tabular}

TABLE 3: The effects of various parameters on Sherwood number (Sh) when $t=1, R=0.1, \mathrm{Gr}=1, M=K=1, \mathrm{Gm}=2$.

\begin{tabular}{llccc}
\hline $\operatorname{Pr}$ & $\gamma$ & Sc & Sr & Sh \\
\hline 0.71 & 1 & 0.6 & 2 & 1.36 \\
$\mathbf{1}$ & 1 & 0.6 & 2 & 0.84 \\
0.71 & $\mathbf{2}$ & 0.6 & 2 & 1.9 \\
0.71 & 1 & $\mathbf{1}$ & 2 & 0.96 \\
0.71 & 1 & 0.6 & $\mathbf{3}$ & 1.64 \\
\hline
\end{tabular}

Tables 1-3. In all these tables, it is noted that the comparison of each parameter is made with first row in the corresponding table. It is found from Table 1 that the effect of each parameter on the skin friction shows quite opposite effect to that of the velocity of the fluid. For instance, when we increase the magnetic parameter $M$, the skin friction increases, as we observed previously velocity decreases. It is observed from Table 2 that Nusselt number increases with increasing values of Prandtl number Pr, whereas it decreases when the radiation parameter $R$ is increased. From Table 3, we observed that Sherwood number goes on increasing with increasing $\gamma$ and Sc, but the trend reverses for large values of Pr and Sr.

\section{Conclusions}

The exact solutions for the unsteady free convection MHD flow of an incompressible viscous fluid passing through a porous medium and heat and mass transfer are developed by using Laplace transform method for the uniform motion of the plate. The solutions that have been obtained are displayed for both small and large times which describe the motion of the fluid for some time after its initiation. After that time the transient part disappears and the motion of the fluid is described by the steady-state solutions which are independent of initial conditions. The effects of different parameters such as Grashof number Gr, Hartmann number $M$, porosity 
parameter $K$, Prandtl number $\operatorname{Pr}$, radiation parameter $R$, Schmidt number Sc, Soret number Sr, and chemical reaction parameter $\gamma$ on the velocity distributions, temperature, and concentration profiles are discussed. The main conclusions of the problem are listed below.

(i) The effects of Hartmann number and porosity parameter on velocity are opposite.

(ii) The velocity increases with increasing values of $K$, Gr, and $t$, whereas it decreases for larger values of $M$ and $\operatorname{Pr}>1$.

(iii) The temperature and thermal boundary layer decrease owing to the increase in the values of $R$ and Pr.

(iv) The fluid concentration decreases with increasing values of $\gamma$ and Sc, whereas it increases when Sr and Pr are increased.

\section{Future Recommendations}

Convective heat transfer is a mechanism of heat transfer occurring because of bulk motion of fluids and it is one of the major modes of heat transfer and is also a major mode of mass transfer in fluids. Convective heat and mass transfer takes place through both diffusion-the random Brownian motion of individual particles in the fluid-and advection, in which matter or heat is transported by the larger-scale motion of currents in the fluid. Due to its role in heat transfer, natural convection plays a role in the structure of Earth's atmosphere, its oceans, and its mantle. Natural convection also plays a role in stellar physics. Motivated by the investigations especially those they considered the exact analysis of the heat and mass transfer phenomenon (see for example, Seth et al. [22], Toki [24], Das and Jana [26], Osman et al. [27], Khan et al. [28], and Sparrow and Cess [29]) and the extensive applications of non-Newtonian fluids in the industrial manufacturing sector, it is of great interest to extend the present work for nonNewtonian fluids. Of course, in non-Newtonian fluids, the fluids of second grade and Maxwell form the simplest fluid models where the present analysis can be extended. However, the present study can also by analyzed for Oldroyd-B and Burger fluids. There are also cylindrical and spherical coordinate systems where such type of investigations are scarce. Of course, we can extend this work for such type of geometrical configurations.

\section{Acknowledgment}

The authors would like to acknowledge the Research Management Centre, UTM, for the financial support through vote numbers 4F109 and 02H80 for this research.

\section{References}

[1] A. Raptis, "Flow of a micropolar fluid past a continuously moving plate by the presence of radiation," International Journal of Heat and Mass Transfer, vol. 41, no. 18, pp. 2865-2866, 1998.
[2] Y. J. Kim and A. G. Fedorov, "Transient mixed radiative convection flow of a micropolar fluid past a moving, semi-infinite vertical porous plate," International Journal of Heat and Mass Transfer, vol. 46, no. 10, pp. 1751-1758, 2003.

[3] H. A. M. El-Arabawy, "Effect of suction/injection on the flow of a micropolar fluid past a continuously moving plate in the presence of radiation," International Journal of Heat and Mass Transfer, vol. 46, no. 8, pp. 1471-1477, 2003.

[4] H. S. Takhar, S. Roy, and G. Nath, "Unsteady free convection flow over an infinite vertical porous plate due to the combined effects of thermal and mass diffusion, magnetic field and Hall currents," Heat and Mass Transfer, vol. 39, no. 10, pp. 825-834, 2003.

[5] M. S. Alam, M. M. Rahman, and M. A. Samad, "Dufour and Soret effects on unsteady MHD free convection and mass transfer flow past a vertical porous plate in a porous medium," Nonlinear Analysis. Modelling and Control, vol. 11, no. 3, pp. 217-226, 2006.

[6] R. C. Chaudhary and J. Arpita, "Combined heat and mass transfer effect on MHD free convection flow past an oscillating plate embedded in porous medium," Romanian Journal of Physics, vol. 52, no. 5-7, pp. 505-524, 2007.

[7] M. Ferdows, K. Kaino, and J. C. Crepeau, "MHD free convection and mass transfer flow in a porous media with simultaneous rotating fluid," International Journal of Dynamics of Fluids, vol. 4, no. 1, pp. 69-82, 2008.

[8] V. Rajesh, S. Vijaya, and k. varma, "Heat Source effects on MHD flow past an exponentially accelerated vertical plate with variable temperature through a porous medium," International Journal of Applied Mathematics and Mechanics, vol. 6, no. 12, pp. 68-78, 2010.

[9] V. Rajesh and S. V. K. Varma, "Radiation effects on MHD flow through a porous medium with variable temperature or variable mass diffusion," Journal of Applied Mathematics and Mechanics, vol. 6, no. 1, pp. 39-57, 2010.

[10] A. A. Bakr, "Effects of chemical reaction on MHD free convection and mass transfer flow of a micropolar fluid with oscillatory plate velocity and constant heat source in a rotating frame of reference," Communications in Nonlinear Science and Numerical Simulation, vol. 16, no. 2, pp. 698-710, 2011.

[11] U. N. Das, S. N. Ray, and V. M. Soundalgekar, "Mass transfer effects on flow past an impulsively started infinite vertical plate with constant mass flux-an exact solution," Heat and Mass Transfer, vol. 31, no. 3, pp. 163-167, 1996.

[12] R. Muthucumaraswamy, P. Ganesan, and V. M. Soundalgekar, "Heat and mass transfer effects on flow past an impulsively started vertical plate," Acta Mechanica, vol. 146, no. 1-2, pp. 18, 2001.

[13] T. Hayat and Z. Abbas, "Heat transfer analysis on the MHD flow of a second grade fluid in a channel with porous medium," Chaos, Solitons and Fractals, vol. 38, no. 2, pp. 556-567, 2008.

[14] M. M. Rahman and M. A. Sattar, "Magnetohydrodynamic convective flow of a micropolar fluid past a continuously moving vertical porous plate in the presence of heat generation/absorption," Journal of Heat Transfer, vol. 128, no. 2, pp. 142-152, 2006.

[15] Y. J. Kim, "Unsteady MHD convection flow of polar fluids past a vertical moving porous plate in a porous medium," International Journal of Heat and Mass Transfer, vol. 44, no. 15, pp. 2791-2799, 2001.

[16] M. Kaviany, "Boundary-layer treatment of forced convection heat transfer from a semi-infinite flat plate embedded in porous 
media," Journal of Heat Transfer, vol. 109, no. 2, pp. 345-349, 1987.

[17] K. Vafai and C. L. Tien, "Boundary and inertia effects on flow and heat transfer in porous media," International Journal of Heat and Mass Transfer, vol. 24, no. 2, pp. 195-203, 1981.

[18] B. K. Jha and C. A. Apere, "Combined effect of hall and ion-slip currents on unsteady MHD couette flows in a rotating system," Journal of the Physical Society of Japan, vol. 79, Article ID 104401, 2010.

[19] G. Mandal, K. K. Mandal, and G. Choudhury, "On combined effects of coriolis force and hall current on steady MHD couette flow and hear transfer," Journal of the Physical Society of Japan, vol. 51, no. 1982, 2010.

[20] M. Katagiri, "Flow formation in Couette motion in magnetohydro-dynamics," Journal of the Physical Society of Japan, vol. 17, pp. 393-396, 1962.

[21] R. C. Chaudhary and J. Arpita, "An exact solution of magnetohydrodynamic convection flow past an accelerated surface embedded in a porous medium," International Journal of Heat and Mass Transfer, vol. 53, no. 7-8, pp. 1609-1611, 2010.

[22] G. S. Seth, M. S. Ansari, and R. Nandkeolyar, "MHD natural convection flow with radiative heat transfer past an impulsively moving plate with ramped wall temperature," Heat Mass and Transfer, vol. 47, pp. 551-561, 2011.

[23] C. J. Toki and J. N. Tokis, "Exact solutions for the unsteady free convection flows on a porous plate with time-dependent heating," Zeitschrift fur Angewandte Mathematik und Mechanik, vol. 87, no. 1, pp. 4-13, 2007.

[24] C. J. Toki, "Free convection and mass transfer flow near a moving vertical porous plate: an analytical solution," Journal of Applied Mechanics, Transactions ASME, vol. 75, no. 1, Article ID 0110141, 2008.

[25] K. Das, "Exact solution of MHD free convection flow and mass transfer near a moving vertical plate in presence of thermal radiation," African Journal of Mathematical Physics, vol. 8, pp. 2941, 2010.

[26] K. Das and S. Jana, "Heat and mass transfer effects on unsteady MHD free convection flow near a moving vertical plate in porous medium," Bulletin of Society of Mathematicians, vol. 17, pp. 15-32, 2010.

[27] A. N. A. Osman, S. M. Abo-Dahab, and R. A. Mohamed, "Analytical solution of thermal radiation and chemical reaction effects on unsteady MHD convection through porous media with heat source/sink," Mathematical Problems in Engineering, vol. 2011, Article ID 205181, 18 pages, 2011.

[28] I. Khan, F. Ali, S. Shafie, and N. Mustapha, "Effects of Hall current and mass transfer on the unsteady MHD flow in a porous channel," Journal of the Physical Society of Japan, vol. 80, no. 6, Article ID 064401, pp. 1-6, 2011.

[29] E. M. Sparrow and R. D. Cess, Radiation Heat Transfer, Hemisphere, Washington, DC, USA, 1978. 


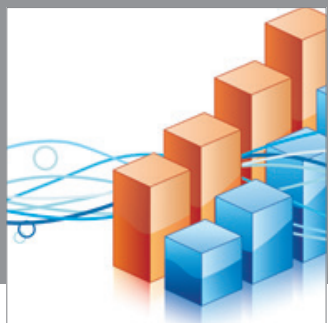

Advances in

Operations Research

mansans

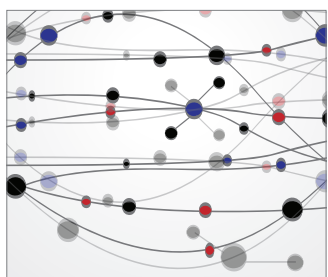

The Scientific World Journal
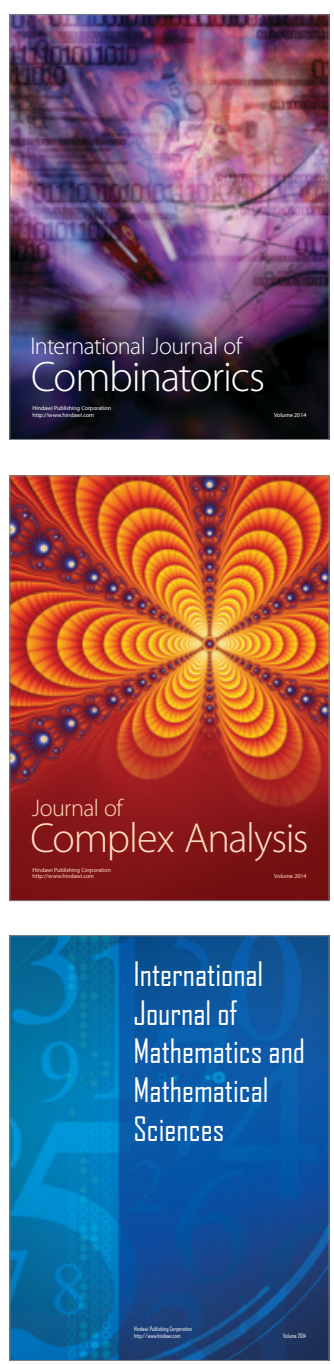
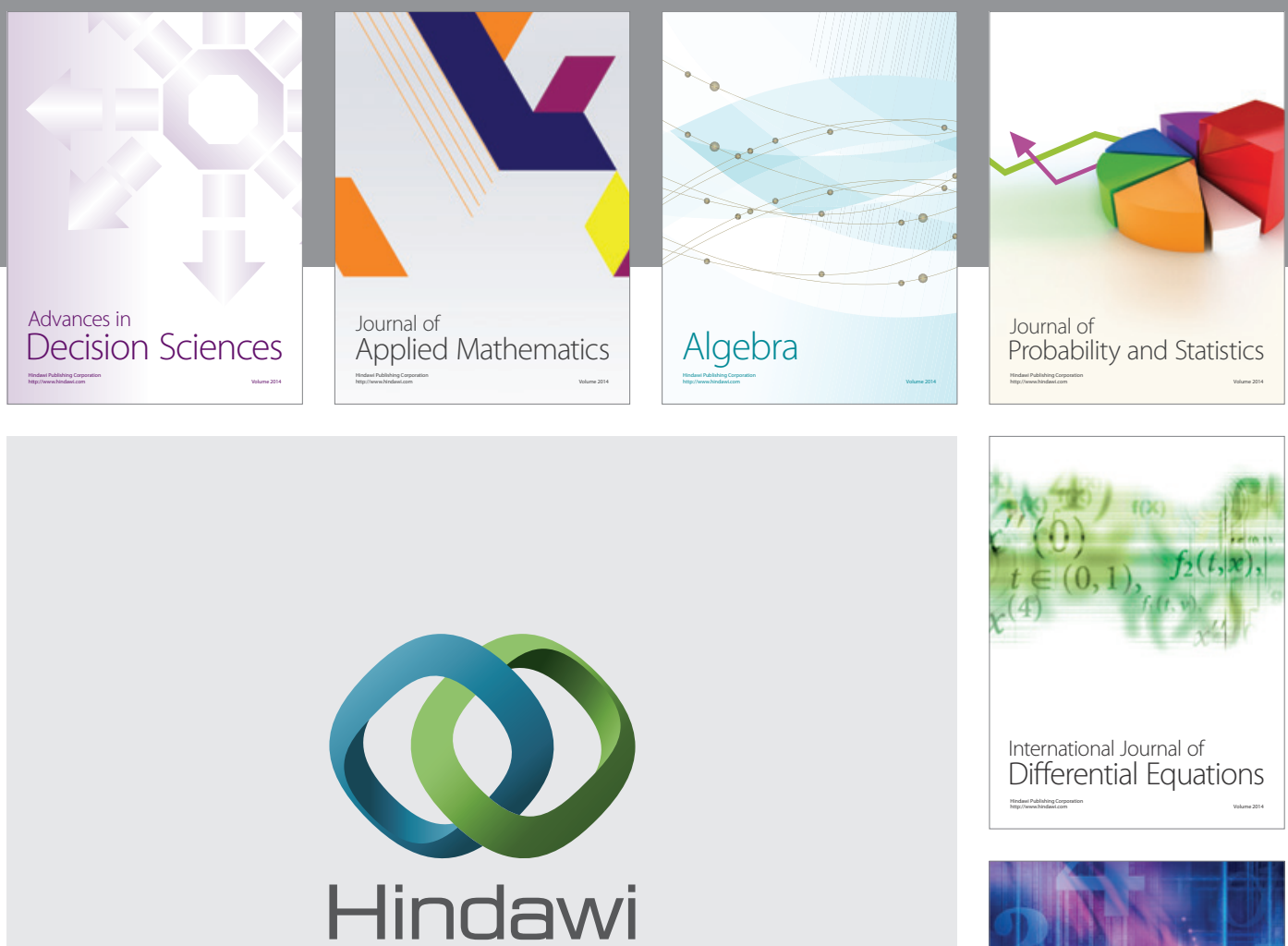

Submit your manuscripts at http://www.hindawi.com
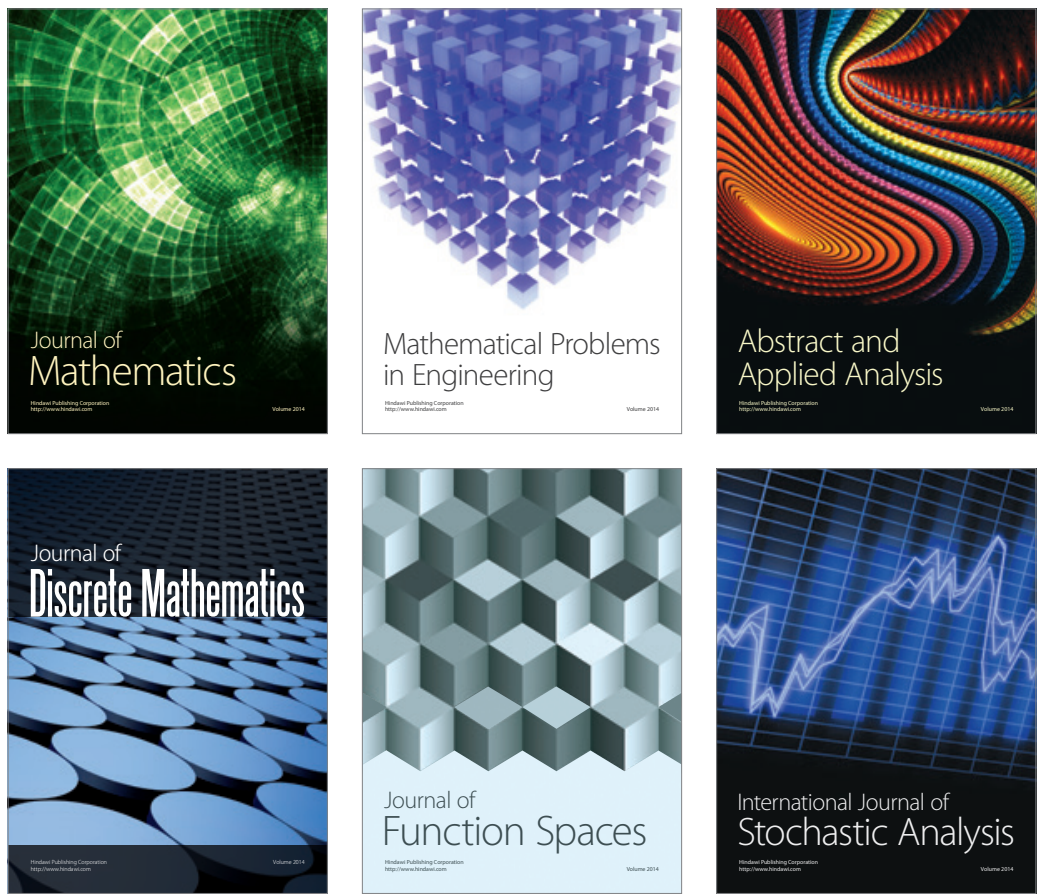

Journal of

Function Spaces

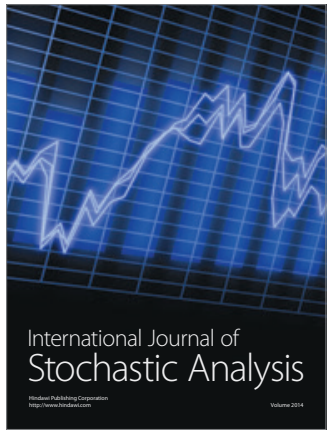

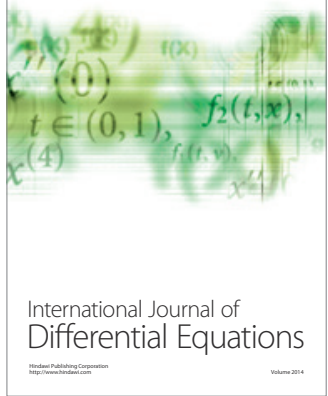
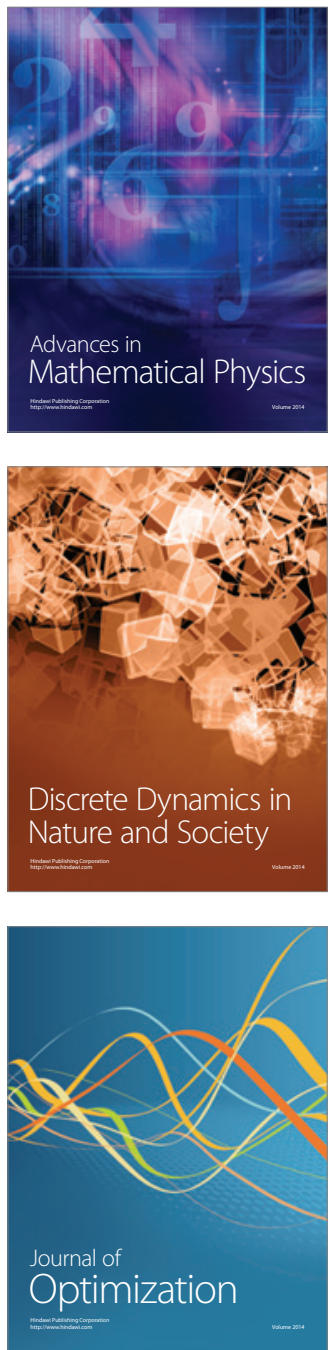